\title{
Arborescences
}

Revue d'études françaises

\section{Un " gai savoir » : stratégies du rire dans les lettres d'une érudite des Lumières}

\section{Mathilde Chollet}

Numéro 9, décembre 2019

La lettre érudite. Nouvelles recherches sur la communication savante à l'époque moderne $\left(\mathrm{XVI}^{\mathrm{e}}-\mathrm{XVIII}{ }^{\mathrm{e}}\right.$ siècles $)$

URI : https://id.erudit.org/iderudit/1068278ar

DOI : https://doi.org/10.7202/1068278ar

Aller au sommaire du numéro

Éditeur(s)

Département d'études françaises, Université de Toronto

ISSN

1925-5357 (numérique)

Découvrir la revue

Citer cet article

Chollet, M. (2019). Un " gai savoir » : stratégies du rire dans les lettres d’une érudite des Lumières. Arborescences, (9), 106-120.

https://doi.org/10.7202/1068278ar

\section{Résumé de l'article}

Entre 1753 et 1755, la châtelaine éclairée Henriette Edme des Rouaudières écrit à son ami le parlementaire exilé Louis Angran d'Allerai. Dans les 35 lettres conservées de leur correspondance, elle mobilise le bel esprit quasi systématiquement en l'associant aux échanges savants qu'elle entretient avec son correspondant. Belles lettres, physique, théologie, philosophie ou politique sont l'occasion de jeux de mots, clins d'oeil, remarques badines. Entre rire érudit et érudition riante, les lettres d'Henriette font état d'une remarquable ambigüité : écrire à Angran revêt un enjeu sérieux pour elle, mais n’empêche pas la légèreté du propos, une conciliation que seule la lettre permet. Tout cela éclaire les stratégies discursives complexes de cette érudite isolée : valorisation de soi par l'esprit, trait classique des élites françaises ; banalisation de l'échange érudit, de ce fait plus acceptable sous une plume féminine ; réduction des distances qui existent entre elle et son prestigieux interlocuteur. À la fois mondain, savant et politique, le discours épistolaire d'Henriette est un bel exemple de l'agentivité féminine au XVIII ${ }^{\mathrm{e}}$ siècle.
Tous droits réservés @ Département d'études françaises, Université de Toronto, 2020
Cedocument est protégé par la loi sur le droit d'auteur. L'utilisation des services d'Érudit (y compris la reproduction) est assujettie à sa politique d'utilisation que vous pouvez consulter en ligne.

https://apropos.erudit.org/fr/usagers/politique-dutilisation/ 


\section{SOMMAIRE}

1 Sébastien Drouin, Université de Toronto

Camelia Sararu, Université de Toronto

Introduction

10 Myron McShane, Centre d'études sur la Réforme et la Renaissance, Université de Toronto Une lettre de Jean Dorat sur l'œuvre de Nonnos

31 Benoît Autiquet, Université de Bâle

Ce que la lettre familière fait au discours médical.

Une lecture de la lettre XIX, 16 des Lettres de Pasquier (1619)

49 Guillaume Bazière, Université Paris Nanterre

Présence érudite et savoirs politiques dans la correspondance du Grand Condé

68 Vanezia Pârlea, Université de Bucarest

Lettres d'Orient : échanges épistolaires en contexte interculturel dans les Mémoires du chevalier d'Arvieux

81 Yves Moreau, Université de Lyon

"Qualche novità litteraria»: la correspondance

entre Jacob Spon (1647-1685) et Antonio Magliabechi (1633-1714)

95 Corinne Marchal, Université de Franche-Comté

La dynamique de la circulation des savoirs et de leurs matériaux dans les échanges épistolaires entre Jean-Baptiste Boisot et Paul Pellisson-Fontanier (1674-1693)

106 Mathilde Chollet, Le Mans Université

Un «gai savoir»: stratégies du rire dans les lettres

$d$ 'une érudite des Lumières 


\title{
Un «gai savoir»: stratégies du rire dans les lettres d'une érudite des Lumières
}

\author{
Mathilde Chollet, Le Mans Université
}

\section{Résumé}

Entre 1753 et 1755, la châtelaine éclairée Henriette Edme des Rouaudières écrit à son ami le parlementaire exilé Louis Angran d'Allerai. Dans les 35 lettres conservées de leur correspondance, elle mobilise le bel esprit quasi systématiquement en l'associant aux échanges savants qu'elle entretient avec son correspondant. Belles lettres, physique, théologie, philosophie ou politique sont l'occasion de jeux de mots, clins d'œil, remarques badines. Entre rire érudit et érudition riante, les lettres d'Henriette font état d'une remarquable ambigüité: écrire à Angran revêt un enjeu sérieux pour elle, mais n'empêche pas la légèreté du propos, une conciliation que seule la lettre permet. Tout cela éclaire les stratégies discursives complexes de cette érudite isolée: valorisation de soi par l'esprit, trait classique des élites françaises; banalisation de l'échange érudit, de ce fait plus acceptable sous une plume féminine; réduction des distances qui existent entre elle et son prestigieux interlocuteur. À la fois mondain, savant et politique, le discours épistolaire d'Henriette est un bel exemple de l'agentivité féminine au XvIII ${ }^{\mathrm{e}}$ siècle.

Femme de plume, la châtelaine vendômoise Henriette Edme (1719-1784) a laissé des manuscrits intimes; de curieuses Pensées errantes suivies d'un roman, les Lettres d'un Indien (Paris, Hardy, 1758); des traductions inédites de Térence; ainsi qu'une correspondance active dont ne nous sont parvenues que quelques bribes (Chollet 2016; Chollet et Krief 2017). Dès l'enfance, elle est friande d'histoire, de morale, de belles lettres; plus tard, elle se passionne pour la science moderne, la théologie, la philosophie, la politique. Autant de matières réservées aux hommes par tradition et qui constituent, au $\mathrm{xVIII}^{\mathrm{e}}$ siècle, le champ d'une érudition féminine. Les qualités de plume de cette amatrice éclairée sont reconnues par les critiques comme par ses proches (Chollet et Krief 2017: 98), et, si elle n'est pas considérée comme un bel esprit ${ }^{1}$, le rire et le sourire érudits sont présents dans ses écrits. Henriette Edme revendique le droit des femmes à accéder au savoir; or, dans le corpus de ses textes, les 35 lettres adressées à Louis-Alexandre Angran d'Alleray (1713-1801) entre 1753 et 1755 attirent particulièrement l'attention par leur qualité érudite ${ }^{2}$, Henriette y manifestant des connaissances remarquables dans les domaines du savoir précités. À cette époque, Henriette Edme est trentenaire et elle vit seule avec son tuteur - son beau-père de 70 ans - dans un château reculé entre Maine et Vendômois. La question de son avenir imminent (un mariage ou une vie dans la dépendance de ses frères) est sous-jacente dans ses écrits du moment et taraude la jeune femme. C'est dans ce contexte qu'elle entretient cet échange érudit avec Angran, célibataire de son âge, son suzerain, voisin et ami d'enfance, parlementaire janséniste,

1. Voir la critique de ses Pensées errantes dans les Mémoires de Trévoux: «Le bon esprit est plus le lot de cette personne que le bel esprit» («Article LXXX. Nouvelles Littéraires»: 1911).

2. Il s'agit de microfilms de copies pour partie manuscrites, pour partie tapées à la machine, où l'orthographe et la ponctuation originales ont été majoritairement conservées. Afin d'alléger la lecture de cet article, toutes les citations de ce corpus (Des Rouaudières, Lettres à Louis-Alexandre Angran d'Alleray) seront simplement suivies des dates des lettres dont elles sont extraites. 
de surcroît exilé par le pouvoir royal durant la majeure partie de cet échange! Alors, pourquoi s’engaget-elle en riant dans cet échange érudit? Y a-t-il vraiment de quoi rire?

Henriette valorise ce savoir enjoué au cours du paradoxal XviII e siècle, à la fois "âge d'or du rire " (Richardot 2002: 9) et siècle des philosophes qui accusent les rieurs d'aller à l'encontre des progrès des Lumières (de Baecque 2000: 107 et suivantes). Mais c'est aussi un moment de «redéfinition du rire» (Richardot 2002: 10) et de son rapport au savoir, que reflètent les lettres envoyées par Henriette à Angran, où le rire, moderne, assume diverses fonctions face à sa propre érudition. La position d'Henriette Edme est, elle aussi, paradoxale: soucieuse de son identité nobiliaire dans une société conservatrice, elle a néanmoins à cœur d'être acceptée comme femme qui pense. Afin de concilier ces contraires sans tensions ${ }^{3}$, quoi de mieux que le rire?

Si la "gaieté de l'érudition» (Pomeau 1971: 192), le rire féminin (Pillet 2000) et les correspondances entre femmes et hommes (Whatley 1997; Bray 2001) ont intéressé les chercheurs en histoire et en littérature, nous nous trouvons au croisement de trois champs d'étude rarement abordés ensemble dans le XVIII ${ }^{\mathrm{e}}$ siècle français: l'histoire des femmes, l'histoire des savoirs et de leur diffusion, et l'histoire du rire (Reynolds 2010: 293-294). Or, puisque la lettre est un des moyens d'expression reconnus aux femmes du temps, elle est, pour le chercheur, un terrain privilégié d'observation des stratégies féminines de valorisation de soi. Henriette joue avec l'esprit, puissant outil dans sa correspondance avec son ami Angran. Seront donc évoquées les conditions de l'échange épistolaire, l'ambiguïté des usages du rire dans les lettres érudites d'Henriette et la fragilité de son rire, qui répond à la fragilité de sa situation et du moment crucial de liberté que cet échange représente.

\section{Les plaisantes conditions d'un échange érudit}

Henriette Edme a su profiter de l'exil de son ami pour mettre en valeur son savoir. Elle parvient à faire de leur correspondance un échange érudit et aimable par sa maitrise du genre épistolaire, grâce à ses lectures et au talent de sa plume.

\subsection{Un échange érudit incongru}

Les conditions de l'échange épistolaire et la forme de la lettre elle-même prêtent à rire. D'abord, alors que l'éloignement dû à l'exil de l'un et à la condition de l'autre commanderait tristesse et chagrin suivant les conventions épistolaires, la première lettre d'Henriette annonce une position inverse: «Votre exil me paraît si agréable, que je n'ose plus en souhaiter la fin» (11 juillet 1753).

Mais le rire provient aussi de l'anomalie que constitue par principe cet échange, au point que l'on pourrait considérer ces lettres comme des lettres burlesques. En effet, le contenu est sérieux: les deux correspondants discutent lectures savantes et actualité politique. Le contexte aussi est sérieux: Louis Angran d'Alleray, conseiller à la quatrième chambre des enquêtes du Parlement de Paris, fait partie des premiers parlementaires à avoir dénoncé en 1752 les refus de sacrements du curé de Saint-Étiennedu-Mont. Dans la querelle entre le pouvoir royal et le Parlement, il fait partie des magistrats les plus engagés et est exilé à Bourges en mai 1753, avec 28 autres conseillers parisiens. Là, il participe peu aux activités mondaines et vit l'exil comme un emprisonnement. Il fait partie des austères «enragés», toujours vêtus de noir et activant leur réseau pour défendre leur cause auprès de l'opinion publique

3. «Je ne désire que la paix, [...] cette tranquillité, le seul objet de mon ambition» (5 juin 1754). 
(Maire 2010). Henriette Edme est une voisine d'enfance et une des vassales d'Angran, relevant de son fief de Saint-Agil; en tant que mondaine, elle se présente comme un relais efficace de ses idées dans leur région car elle fréquente les bonnes familles des élites locales. De plus, son réseau de correspondants s'étend à la France de l'Ouest, à Paris et aux Caraïbes. L'on y trouve des personnages intéressants pour Angran, dont un lieutenant civil et criminel ainsi qu' un gouverneur. La gravité de la situation est cependant bouleversée dans les lettres par des éléments qui tendent à la diminuer et d'où surgit par conséquent le burlesque.

$\mathrm{Si}$, par bienséance, Henriette reconnaît le peu d'attirance de son correspondant - et le sien pour le comique («La plaisanterie n'est pas votre Elément, ce n'est pas trop le mien non plus» [8 août 1753]), elle se charge ainsi, d'un docte clin d'œil, d'égayer l'échange: «Vous avez mêlé seria nugis ${ }^{4}$ pour faire honneur à votre exil, et moy je vais tâcher de mêler un peu nugx lamentia 5 pour que ma lettre ne vous paraisse pas une jérémiade» (6 février 1754). En vérité, elle n’a que peu d'efforts à fournir, car le premier sujet de sa première lettre conservée, datée du 11 juillet 1753, est une réponse à des «folies» d'Angran et à une description de son ménage de Bourges qui ont "fort amusé» l'épistolière. Plus loin, il fait selon elle «des réflexions fort justes, et très plaisantes sur cette lettre de rappel qui prolonge [son] exil, et [le] fait ensuite errer pendant onze jours" (24 août 1754), ce qui montre que le parlementaire est soucieux de distraire sa correspondante en dédramatisant le sérieux de l'exil, tout en mettant à profit leurs références bibliques communes. Il n'est donc pas surprenant de lire au long de la correspondance que les deux amis se taquinent: Henriette dénonce une «malice» d'Angran le 29 septembre 1753, ou bien feint d'être froissée ("Vous vous moquez un peu de moy» [17 juillet 1754]) lorsque son interlocuteur commente ses efforts pour mettre son nom en anagramme latine. Elle aussi se moque du sérieux de sa fonction tout en mettant en avant leurs connaissances communes. En l'appelant le «cher censeur» (5 juin 1754, deux occurrences) ou le "cher sénateur» (22 septembre 1754, trois occurrences), elle rappelle d'abord à Angran que la fonction qu'il occupe ne peut distendre leur amitié. Elle fait ensuite référence à son propre goût pour l'histoire romaine et enfin elle le gronde pour l'avoir jugée trop légèrement ( Non, mon cher Censeur, non, je ne suis pas si frivole que vous l'imaginez»)! Même l'exil commandé par le pouvoir royal devient comique quand Henriette s'en sert pour se moquer des défauts de son correspondant:

Sûrement vous ne devez pas craindre, que je vous prenne pour un grand parleur, je n'ai garde; il vous est arrivé bien des fois de me répondre par des «homs» qui vous auroient fait battre si je l'eusse osé; vous ne feriez pas cela présentement, quand on a été quatorze ou quinze mois en garnison, cela forme bien la jeunesse, si vous n'en revenez pas petit maître il faudra vous en sçavoir gré. (24 juillet 1754)

Le burlesque atteint son paroxysme dans la lettre du Nouvel An, occasion d'un concours d'extravagance entre les deux amis, où l'épistolière se livre à une digression hypothétique:

Je me souviens aussi Monsieur, de la longue épître dont je vous assassinai l'année passée, c'était un peu votre faute, vous m'aviez mandé que vous vous attendiez à un compliment singulier de ma part, cela me jeta un embarras dans l'esprit dont je me vangeai en vous disant des folies, et j'avais projetté de vous prévenir cette année en vous faisant le même discours, que je m'attendais à un compliment extraordinaire de votre part, [...] je n'ai pas eu le courage de vous faire cette petite niche, peutêtre que c'est un bonheur pour moy car cela aurait pu produire sur vous un effet tout contraire, et

4. Du sérieux dans le frivole.

5. Du frivole dans les plaintes. 
racourcir votre épître, vous auriez pensé: "Que diantre veut-elle que je luy dise?» Et vous ne vous seriez jamais souvenu, que c'était un retour. ( ${ }^{\mathrm{er}}$ janvier 1755$)$

Un des rituels d'entrée dans la lettre qui dispose à l'enjouement de l'échange érudit est le cliché des lenteurs de la poste. Quand une lettre passe peu de temps sur la route, l'épistolière a "envie d'en écrire des remerciments, à ce gros monsieur de Monregars» (27 février 1754), contrôleur général des postes et relais de France et personnage lié par mariage à la famille Angran d'Alleray: Henriette n'épargne personne. Même l'éloignement du rappel des exilés est l'occasion de rire, car «il n’y a que Mrs des postes qui gagnent à cela, et ils s'affligeront de la fin de votre exil» (6 février 1754). Le coût élevé, la lenteur de la poste aux lettres et la possible surveillance du courrier des exilés sont des obstacles que la jeune femme franchit allègrement, car elle et Angran disposent d'autres intermédiaires ${ }^{6}$.

Pour leur part, les formules de fin empruntent aux correspondances antiques et aux lettres savantes quand Henriette quitte son destinataire d'un «Valere. Vale, vale, et salve» ou d'un «Vale charissime, Cura ut valeas ${ }^{7}$. Du début à la fin de la lettre, Henriette mêle le sérieux et le frivole dans l'objectif de faire oublier à Angran les peines de l'exil, mais aussi dans l'intention de se mettre en avant comme latiniste érudite et de remettre en cause la hiérarchie existant entre elle et son correspondant. Cette mise à niveau des deux épistoliers est la condition nécessaire pour entamer un véritable échange érudit, d'autant qu'Henriette n'a rien à envier à la culture livresque du noble magistrat.

\subsection{La culture de deux rieurs}

En août 1755, à l'occasion du mariage d'Henriette, un inventaire de ses biens est dressé (Riel: 1755), dans lequel le détail de la bibliothèque n'est pas oublié. De même, on connaît les livres à disposition de Louis Angran par l'inventaire de son château de Saint-Agil et par celui de son appartement parisien (Leroy: 1733 et Boulard: 1801). Ces trois documents ne disent pas ce que nos deux correspondants ont lu; cependant, ils permettent d'apprécier les auteurs d'ouvrages savants et les domaines du savoir dont ils ont sans doute entendu parler. Il s'agit de bibliothèques érudites relativement classiques, malgré l'absence de secrétaires ou d'ouvrages présentant différents modèles de lettres. Chez la demoiselle Edme, qui rêve de belles bibliothèques et aimerait lire tous les livres (Chollet et Krief 2017: 301), dominent l'histoire, la piété, la morale, la littérature moderne et le souci du beau langage. Chez Angran, Anciens et Modernes, livres de droit, d'histoire, de piété et de théologie, ouvrages relatifs aux finances, au commerce, aux coutumes, à la guerre, à l'architecture et aux «antiquités» s'accompagnent d'ouvrages mauristes de numismatique et de diplomatique qui supposent tous, de la part du parlementaire, un goût pour l'érudition.

Mais les livres présents dans ces bibliothèques sont aussi des ouvrages de divertissement, où les deux correspondants ont sans doute puisé leur inspiration comique. Le Voyage de Bachaumont et Chapelle que possède $\mathrm{M}^{\mathrm{lle}}$ Edme est un récit de voyage léger et divertissant en même temps qu'une critique subtile de l'absolutisme (Rauline 2007). Les romans épistolaires de sa bibliothèque sont aussi des livres plaisants, badins, voire carrément considérés comme libertins et persifleurs. Il en est ainsi des Lettres de Ninon de Lenclos au marquis de Sévigné, des Lettres de la marquise de $M^{* * *}$ au comte de $R^{* * *}$ de Crébillon fils et des Confessions du comte de ${ }^{* *}$ de Duclos. Et que penser des aventures du

\footnotetext{
6. «Quoy que j’honore beaucoup la poste, je vous suis bien obligée Monsieur, de m’avoir écrit par Boulai, j’ai eu votre lettre trente heures plus tôt, cela vaut la peine d'en être bien aise» (28 mai 1754).

7. «Porte-toi bien», «prends soin de toi», «cordialement»,...
} 
Roland amoureux de Boiardo, dont la dimension comique a été conservée par Lesage, ou encore de la succession de malheurs subis par le picaresque Infortuné Napolitain? Et puisque cette érudite à la plume aguerrie est aussi friande de néologismes, mentionnons qu'elle possède le Dictionnaire néologique à l'usage des beaux esprits du siècle de Desfontaines, ainsi qu'un recueil de bons mots, dont elle peut profiter pour amuser ses interlocuteurs. Hors de sa bibliothèque, dans les Caractères de La Bruyère par exemple, lus avec passion pendant l'adolescence (Chollet et Krief 2017: 205), elle a appris le bon mot et la raillerie qui font mouche. Ses lectures précieuses (Le Grand Cyrus) ont pu inspirer son goût pour le badinage. De plus, Henriette est amatrice de comédies, même si elle fustige la farce grossière (Chollet et Krief 2017: 353-357). Dans sa jeunesse, elle est l'auteure d'une comédie inspirée de Molière (Chollet et Krief 2017: 206-207), puis une érudite traductrice des comédies de Térence. Et puisque Cicéron est une de ses références en matière d'amitié (17 avril 1754), nul doute qu'elle le connaît comme rhéteur au rire redoutable; elle a d'ailleurs pu emprunter ses épîtres et ses œuvres à la bibliothèque parisienne d'Angran. Lui-même, tout austère qu'il soit dans la défense de ses fonctions, n'est pas étranger au rire. Il a hérité des Perroniana et Thuana de son père (un recueil de Pensées judicieuses, bons mots, rencontres agréables et observations curieuses du Cardinal du Perron et de M. le Président de Thou), du roman picaresque Gil Blas (et il a le Quichotte à Paris) et de L'Homme de Cour de Gracián, dont on peut estimer que la dénonciation du ridicule fait écho au rire mordant de son exemplaire des CEuvres de Boileau. Et dans les six volumes de l'Espion Turc inventoriés à Paris, il est probable qu'il trouve l'historiette amusante qu'Henriette consigne dans son journal de 1754 (Chollet et Krief 2017: 282) et bien d'autres encore... Les sources d'inspiration des deux correspondants sont donc des références classiques: Anciens, auteurs du XVI ${ }^{\mathrm{e}}$, du Grand Siècle et contemporains ayant codifié différentes formes du rire "de bon goût» et dont ils reprennent les préceptes, montrant ainsi leur appartenance à un groupe supérieur (Richardot 2002: 96).

\subsection{Les formes du rire chez Henriette Edme épistolière}

L'objectif de ce travail n'est pas d'exposer les ressorts de l'érudition d'Henriette en matière de rire. Dans sa correspondance, la définition du rire s'étend certes sur une large gamme: sourire, esprit, «belle humeur $^{8}$ » (17 octobre 1753), dérision, badinage, plaisanterie, comique, jeux de mots, ironie, boutade... Mais cette définition est forcément incomplète, puisque des formes (dé)passées du rire nous échappent assurément aujourd'hui et que certains passages procédant du rire pour le lecteur actuel n'étaient peut-être pas considérés comme tels dans l'esprit d'Henriette et celui de son correspondant (Le Goff 1997: 449; Andries 2000: 10). Reconnaissons toutefois que la langue de l'épistolière est riante: ses lettres présentent une variété de rires au service de l'écriture érudite.

Mondaine, la jeune femme est soucieuse de la politesse, de la justesse et de la noblesse de son style. En ce sens, pasticher $\mathrm{M}^{\mathrm{me}}$ de Sévigné ("Je vous la donne à deviner en cent, en mille, en cent mille» [24 juillet 1754]) est un moyen facile de valoriser et sa plume d'épistolière et ses lectures. Elle se permet pourtant des légèretés que la lettre familière autorise (Haroche-Bouzinac 1995: 24-25) et sa plume est parfois relâchée. Or, le comique fait partie de ce style familier tel que l'a défini Vaugelas (2009: 67 et 84). Ainsi, comme dans ses manuscrits intimes, les figures de style amusantes jalonnent ses lettres, telle cette métaphore filée qui accompagne un compte-rendu de la lecture de L’Esprit des Lois: «Je me fais un plaisir de le suivre, et de voir comme en marchant toujours, il va droit à son but, quoy qu'il paraisse

8. Cette expression peut se rapprocher de l'«humour ", mot d'origine anglaise que la langue française adoptera progressivement au cours du siècle. L'autodérision d'Henriette le confirmerait d'ailleurs. 
biaiser quelquefois» (5 juin 1754), ou ce paradoxe résumant son avis sur l'Essai sur les bienséances oratoires de l'abbé Mallet: "un petit recueil de choses que tout le monde sçait, et auxquelles mille gens n'ont pas fait attention» (17 juillet 1754). Sarcasme et expressions familières s'unissent pour dénoncer une lecture ennuyeuse:

Nous faisons actuellement une belle lecture ce sont les voyages de Spilberg-van-der-does-houtmanwillems verhoven-van groennewagen-janz-hem-vallicshz-hesseti-ez qui sont des hollandais, lesquels ont voyagé partout, et vû tant de choses que c'est pitié; je suis encore en état de vous régaler de quelques noms japonais, longs comme le bras; il n'y a rien de si amusant que cela, ny qui donne moins de pensées; quand j'ai lu pendant deux heures il me semble que j'ai la tête comme une lanterne cependant il n'y en a que 12 volumes, excusez du peu. (18 décembre 1754)

Et même les jeux de mots, bannis eux aussi du beau langage, font forcément l'éloge de sa culture, qu'il s'agisse d'un quiproquo sur Racine:

J'ai eu par mégarde, les cinq volumes de Racine le fils, [...] j'avais prié un bon bénédictin, de m’apporter de Paris, Jean Racine, en petit format; il a cru sans doute, que touttes les racines estoient la même chose, ou qu'on ne les distinguoy qu’à la taille (27 février 1754);

ou du double sens du mot «gravité», quand Angran rentré d'exil se permet «deux gambades dans le salon »: "Vous pourrez très bien avoir perdu l'équilibre de la gravité» (22 septembre 1754), l'excuse-telle. En somme, ces différentes manières de faire rire enrichissent la langue de l'épistolière, à l'image de ses lectures et de ses savoirs. Cependant, si elle est capable de s'affranchir des conventions relatives à la forme des lettres, on peut s'interroger sur l'originalité des idées qu'elle y expose.

\section{L'échange érudit entre conventions et liberté}

Le rire érudit peut être le moyen pour Henriette de se mettre en valeur auprès de son correspondant, tout en respectant les règles de sociabilité et de civilité, qui recommandent aux femmes une conversation à la fois de bon goût et superficielle (Diaz 1998: 136-137). Dans ce cas, rire et convention l'emportent sur l'érudition. Mais les lettres à Angran sont aussi un espace de liberté où Henriette peut réfléchir et s'exprimer sans craindre de déplaire à son correspondant.

\subsection{Rire et savoir au service de l'honnêteté}

Henriette écrit immédiatement après la disparition du noble et joyeux Régiment de la calotte, qui défend tant la frivolité du bel esprit qu'un «savoir érudit du rire» (de Baecque 1997: 482), et il est intéressant de constater qu'elle en suit la discipline. Évoluant dans le même milieu social que les calotins, son rire en respecte les conventions et dénonce les excès et le ridicule. On sait que les traités de conversation et les secrétaires conseillent aux épistoliers "une désinvolte négligence» (HarocheBouzinac 1995: 58) afin d'éviter l'accusation de pédanterie. Les lettres d'Henriette respectent ces préceptes. 
En société, l'honnêteté oblige à ne pas considérer les occupations érudites comme sérieuses, au risque sinon de verser dans le ridicule ${ }^{9}$. C'est pourquoi $M^{\text {lle }}$ Edme, pourtant bonne latiniste, fait preuve d'autodérision quand elle commente ses résultats anagrammatiques peu satisfaisants:

Vous avez bien de la bonté d'être content de votre anagramme, il fallait bien que vous fussiez Lux ou Rex car que faire de cet $x$ : j'avais mis d'abord, Rex Cordis. Mais cela employait bien des lettres et il restait tous ces chiens d'A dont je ne sçavais que faire, ainsi je vous ai fait Lux. (17 juillet 1754)

De plus, afin de réduire la distance physique qui sépare les deux correspondants, la lettre doit imiter les règles de la conversation (Grassi 1981 : 174). Les lettres d'Henriette, tout érudites qu'elles soient, se doivent donc d'avoir une dimension amusante ${ }^{10}$. Sous la plume d'Henriette, écrire à Angran est donc distrayant (16 décembre 1753), c'est "un plaisir» (27 février et 7 août 1754) ou encore "une récréation» (29 avril 1754); et elle se justifie de ses «niaiseries» par l'intention d' "amuser » Angran (29 avril 1754). Rire est un signe de savoir-vivre chez ces notables pour qui les occasions de sociabilité sont centrales et où chacun a le devoir de distraire l'autre. En ajoutant du «sel» (8 août 1753), du piquant à la conversation, les deux épistoliers évitent la fadeur dans leur échange.

Mais ce sel sert aussi à l'assaisonner d'une pointe de raillerie, l'érudition entrant alors au service du bon mot. Par exemple, à propos d'une de leurs amies:

On dit qu'elle est actuellement attachée, accrochée, à Mr de Champeron, tout saintement, tout spirituellement, mais d'une force invincible je pense que c'est par un effet de cette vertu magnétique dont je vous parlais l'autre jour, il ne scaurait m'entrer dans la tête qu'il y ait d'autres raisons de cette sympathie, car on dit qu'il a bien de l'esprit, enfin donc, le tourbillon de matière subtile qui enveloppe l'un des deux, entraîne l'autre dans son mouvement continuel; je ne sais de quel côté est l'aiman, mais l'attraction est complètte à ce qu'on assure. (30 août 1753)

Notons qu'en se moquant de cette femme, Henriette, dans l'esprit du temps, se moque aussi de la métaphysique du siècle précédent. Le respect des règles de savoir-être est essentiel parmi les élites, d'où l'omniprésence d'un rire de convention dans les lettres d'Henriette, qui ne parvient cependant pas à masquer parfaitement son savoir, inconvenant chez une femme.

Dans les lettres d'Henriette, le souci de faire le bel esprit, de provoquer un rire fidèle aux règles de l'art de plaire, semble dominer. Ainsi, après des débuts où, on l'a vu, chacun tâtonne pour borner l'espace de liberté bienséant, la conversation glisse discrètement et savamment sur le terrain du badinage, comme dans ce jeu des anagrammes:

On trouve que je sçai bien aimer, mais on ne dit point que je doive l'être; enfin c'est la faute de mes parains et maraine, que faire à cela? Ce qu'il y a de bon, c'est que mon anagramme française, est dans le goût de la latine; et si forte, que je n'ai osé la dire, de peur que vous ne la prissiez absolument au pied de la lettre. (29 avril 1754)

9. « $[\mathrm{U}]$ ne femme a grand tort, et n'acquiert que du ridicule lorsqu'elle s'affiche pour savante ou pour bel esprit», déplore Louise d'Épinay dans sa lettre à Galiani du 4 janvier 1771 (Correspondance: II, 26).

10. C'est d'ailleurs ce que recommande l'abbé Mallet dans son Essai sur les bienséances oratoires (qu'a lu Henriette entre le 10 et le 17 juillet 1754, d'après ses lettres à Angran) : «Les Lettres où il ne s'agit que d'érudition, de critique [...] tireront toujours leur principal mérite de la justesse \& de la précision. Mais rien n’empêche qu’on n’y répande une certaine fleur d'élégance et d'urbanité» (II : 146). 
Immédiatement, Henriette qualifie ce développement de «niaiserie» pour rester dans les bornes d'une inoffensive politesse. Le badinage est d'ailleurs présenté à quatre reprises (par l'emploi de l'expression «badinage à part») comme un écart involontaire, qu'il faut excuser. Et c'est parce qu'Angran la suit sur ce terrain qu'elle se sent obligée de répondre le 2 novembre: «Je finirai demain ma lettre à St-Agil avec $\mathrm{M}^{\text {me }}$ d'Alleray que j'embrasserai pour moy et non pour vous; vous êtes bien plaisant? Pour qui me prenez-vous?»; puis que, le lendemain, elle invoque la théorie des climats pour excuser cet emportement ${ }^{11}$. Il n'est donc pas anodin que, lorsque la conversation dévie vers un sujet plus délicat, celui de la nature des liens qui les unissent, tout le développement d'Henriette sur un idéal changement de sexe («Nous serions bien plus libres ensemble, nous pourrions même nous arranger pour demeurer sous le même toit» [26 décembre 1753]) est qualifié du rassurant terme de "badinage», sur lequel les deux correspondants promettent tout de même mutuellement discrétion... Or, même derrière le badinage, ce thème de la métamorphose est «un procédé comique très ancien» (Pillet $2000: 10$ ), basé sur l'impossible et le monde à l'envers, une arme féminine dénonçant les limites assignées aux femmes.

Le rire est donc un moyen pour Henriette de respecter les bienséances, qu'elle infirme pourtant en correspondant avec un homme célibataire à qui elle expose en toute confiance ses savoirs et ses idées. Elle a pourtant tout intérêt à respecter les règles sociales et à user de la plaisanterie badine, si elle souhaite prolonger cet échange particulier avec Louis Angran.

\subsection{Savoir et humour au féminin: protection, plaisir et liberté}

Lautodérision est très présente dans les lettres d'Henriette. Grande lectrice, passant des heures à lire, elle bute pourtant sur Le Grand Cyrus: "J'ai passé courageusement, tout plein de monologues, et de conversations, qui très assurément m’auraient fort ennuyée», avoue-t-elle le 16 décembre 1753. Et même son activité d'épistolière parât rabaissée par son humour quand, par exemple, elle explique à Angran que l'«indifférence me serre le gozier, dessèche ma plume, ainsi je vous conseille d'être bien glorieux de touttes les rapsodies dont je vous accable» (9 juillet 1754); ou lorsqu'elle le plaint d'" estre obligé de lire les volumes qu['elle lui] écri[t], voilà Dieu merci la cinquième page» (6 février 1754). On pourrait n'y voir qu'un signe de respect des conventions épistolaires générales, mais, en procédant ainsi, elle banalise son statut de femme érudite et désamorce le ridicule que représenterait pour son respectable correspondant l'échange avec une femme savante: «Je ne me mêle pas d'instruire les doctes", assure-t-elle le 30 août 1753. Le 17 octobre suivant, elle feint d'ailleurs l'autocensure au nom de la même soi-disant pudeur, en affirmant: "Je ne veux pas vous en dire davantage sur tout cela, car vous prendriez ma lettre pour le journal d'un médecin ", alors que cette déclaration suit un long paragraphe détaillant ses problèmes de santé et ceux de son beau-père! De même, sa courageuse traduction de comédies de Térence, qu'elle poursuit sur plusieurs années en toute discrétion pour ne pas susciter la colère d'un beau-père conservateur, est réduite à une plaisanterie: "J'ai été près d'un an sans rien faire, je crois que j'ai oublié» (17 juillet 1754). Le rire d'Henriette n'est donc pas qu'un rire de consensus, c'est aussi un rire-bouclier.

Elle voit d'ailleurs dans la condition d'Angran exilé un reflet de sa condition de femme érudite: "Je connais mieux que vous encore, ce que c'est de ne pas faire ses volontés: je m'en console parce qu'au moins on n'a pas d'empire sur mon intérieur» (14 août 1754). Cela explique sans doute son désir d'atténuer l'incongruité de leur échange, notamment par le rire. Cependant, si Molière au siècle

11. «Je vous écrivis hier Monsieur, une lettre qui est un peu impertinente l'air y fait un peu, ainsi j'espère que vous m'excuserez en faveur du climat» (3 novembre 1753). 
précédent créait, dans Les Femmes savantes, un monde inversé dans le but de conjuguer moquerie et dénonciation, il est impossible pour Henriette de cautionner cette sorte de rire. Quand elle reproche à Angran de ne pas lui avoir envoyé un discours du président du Parlement, d'une part elle dénonce les clichés sur le retard des provinciaux ( S’il n’y avait pas des affiches pour amuser nous autres fainéants de provinces, je ne l'aurois pas eu en ma possession", 22 septembre 1754), mais d'autre part elle lui rappelle le rôle crucial de diffusion de l'information qu'elle accorde à la lettre. Son apparente autodérision désarme bien les tensions, mais ne ridiculise en aucun cas la figure de la femme qui sait; au contraire, elle la défend. «Vous avez intérêt que je fasse des lectures amusantes, car sans doute je vous en ferai part ", l'épistolière morigène-t-elle Angran le 11 juillet 1753, qui n’a donc d'autre choix que de cautionner les ambitions de sa correspondante.

Les lettres à Angran rompent la solitude et l'isolement d'Henriette. Pour cette femme qui dit l'ennui du quotidien (22 mai 1754) et assure n'avoir de correspondance suivie avec d'autre homme qu'Angran $\left(1^{\text {er }}\right.$ janvier 1755), sans doute cet échange amical représente-t-il un espace de liberté où elle peut exprimer ses idées et trouver «un supplément d'être et un supplément d'âme» (Diaz 1998: 136) ordinairement refusés aux femmes en quête de savoir. Si Henriette justifie son désir d'écrire, de lire et d'apprendre, par l'envie de se divertir et de s'amuser (Chollet et Krief 2017: 430), le lecteur averti ne s'y trompera pas: il s'agit bien d'une stratégie argumentative dont l'objectif est de célébrer, et non de minimiser, sa soif d'érudition. L'habile utilisation du rire dans sa correspondance permet cette ambiguïté. La gaieté de ses lettres et les efforts qu'elle déploie pour respecter les conventions épistolaires dans le but de plaire à son interlocuteur et perpétuer l'échange soulignent le plaisir qu'elle-même prend à écrire. À dix reprises en deux ans, elle évoque le "plaisir» d'échanger avec Angran. Plaisir d'écrire et de discuter avec une "substance qui pense" (3 juillet 1754), mais aussi plaisir de pouvoir enfin s'affirmer comme telle, alors que son beau-père la rappelle aux bienséances attendues ${ }^{12}$. Henriette affecte la soumission aux règles de bonne conduite des femmes en société, dont en réalité elle se moque, puisqu'elle poursuit ses activités intellectuelles et peut en discuter par écrit. Elle bénéficie pour cela d'une conjoncture propice mais exceptionnelle, l'exil d'Angran, dans un contexte plutôt défavorable à son épanouissement par le savoir.

\section{Le rire de l'épistolière: un masque fragile}

Le rire de l'épistolière lui permet de sauver les apparences face à ses proches qui jetteraient un œil sur sa correspondance ou qui en réclameraient lecture. Dans la mesure toutefois où, comme le rappelle Alain Faure, «le rire peut être [...] une arme offensive» (1994: ii), il convient de souligner la polysémie du rire d'Henriette: entre rire savant et rire dramatique, il présente une dimension plus provocatrice, jusqu'à ce que ses lettres n’offrent plus de quoi rire.

\subsection{Le rire: une arme efficace?}

Cette dimension est évidente dès la première lettre adressée à Angran, où l'épistolière non seulement fait valoir sa connaissance de la Bible, mais détourne, sur un ton de badinerie, le sens du verset qu'elle cite au profit des femmes:

12. À titre d'exemple: «Il est retombé dans les douleurs de la gravelle, ainsi j'ai le temps de faire du noir et je l'employe, je me distrais en vous écrivant» (16 décembre 1753). 
Vous souvenez-vous d'une certaine prophétie qui dit, "en ces jours-là sept femmes prendront un homme» notre siècle est bien meilleur vraiment, je serais fâchée d'être venue dans cet autre, l'abondance des rivales ne me plairait pas; je ne crois pas aussi que je m'accommodat mieux, d'une quantité de maris, mais dans cinq on peut choisir. (11 juillet 1753)

Et, dans la même lettre, elle use de jeux de mots pour souligner sa lecture des Entretiens physiques du père Regnault: «Je me suis tirée assez légèrement de la matière subtile, mais j’ai bien pensé embourber dans le mouvement», commence-t-elle, puis: «Pour de repos absolu, il n'en est point, et je le savais à merveilles sans qu'il me le dit». Cette mise en scène d'un savoir décomplexé, que l'on pourrait interpréter comme un critère de distinction de soi si l'on suit Bourdieu (1979: 36-37 et 59), place Henriette en égale de son interlocuteur. En effet, le rire d'Henriette s'empare des champs du savoir masculin, tous susceptibles d'intéresser Angran au vu de sa bibliothèque et de son parcours professionnel: généalogie ${ }^{13}$, médecine et mythologie grecque ${ }^{14}$, métaphysique ${ }^{15}$, sciences modernes ${ }^{16}$ et même théologie ${ }^{17}$. La légèreté du ton a bien l'objectif de distraire le lecteur, tout en lui donnant des nouvelles quotidiennes. Mais l'épistolière y ajoute une dimension érudite par de constants clins d'œil à une variété considérable de sujets savants ou considérés comme trop élevés pour que les femmes puissent y atteindre. Pensons aussi qu'elle fait le choix de traduire du latin des comédies de Térence, et celui d'en informer son interlocuteur préféré: c'est bien qu'elle y trouve un intérêt. Non seulement Angran l'aide ou relit son travail (17 juillet et 18 décembre 1754), mais le dramaturge est réputé difficile à traduire ${ }^{18}$; elle s'est donc attelée à une tâche qui, certes, relève de l'otium, mais qui est avant tout admirable.

Hobbes et, après lui, Bergson, Freud et Bataille voient dans le rire un puissant lien, un moyen de créer une interaction (Richardot 2002: 11-12). Or, la lettre assume déjà ce rôle de création et/ou d'entretien du lien, comme le montre l'appréciation qu'en fait Angran: «Vous me dites que vous aimez mes lettres, $[\ldots]$ je sens là-dessus tout ce que je dois à votre amitié» (7 août 1754). Le rire savant qui ponctue les lettres d'Henriette est donc un moyen de renforcer une connivence, une complicité avec le parlementaire, là où l'amitié et leurs positions respectives ne devraient tolérer que respect et estime. La lettre réduit les distances géographiques, mais y associer de manière inextricable rires et savoirs réduit aussi la distance sociale et intellectuelle existant entre les deux correspondants. Si l'on considère les interdits et le contrôle sévère de l'Ancien Régime imposés par le pouvoir royal et l'Église en matière d'idées - notamment envers les femmes -, on peut estimer que le rire d'Henriette est même un véritable signe d'engagement politique, voire d'une dangereuse liberté (Poirot-Delpech 1994: 9 et 11). Et, effectivement, en écrivant à l'exilé Angran que, dans le conflit l'opposant au Parlement, «la cour ne sçait elle-même, ny ce qu'elle fera, ny ce qu'elle doit faire, pour moy si j'étais le Roy je sçai bien comment je m'en tirerais mais comme il ne prend pas mes avis, je n’irai pas les luy donner» (16 décembre 1753), Henriette flatte certainement son correspondant, mais, à travers l'ambiguïté de son autodérision, elle s'engage en même temps sur le terrain politique.

13. «Si vous me fâchez je vous ferai un arbre généalogique de la maison de Mr le comte des Salles» (17 juillet 1754).

14. «La marmite d'Hipocrate est fort bonne aussi, car elle ressuscite journellement mon beau-père, il est fâcheux pour luy que je n'aye pas le talent de le rajeunir, en le faisant bouillir avec des herbes fines, comme le bonhomme Eson » (2 novembre 1753).

15. «Le beau-père vous complimente, la substance matérielle le porte assez bien» (29 septembre 1753).

16. «Je ne sçai quand nous pourrons diviser la colonne d'air qui est entre nous» (24 août 1754).

17. "Je crois que l'amitié est comme la grâce efficace, elle nous détermine et nous conduit, par des impulsions si douces, que l'on n'a, ny la force, ny la volonté d'y résister" (7 août 1754).

18. "Une tâche bien hardie» selon Diderot (Réflexions sur Térence: 235). 


\subsection{Un rire dramatique et nécessaire}

Ce rire érudit et cette érudition riante que manifestent les lettres d'Henriette ne sont ni une constante, ni un trait naturel. L'épistolière fait sienne la devise de Figaro, déjà défendue par Voltaire (Cotoni 1994), et rit de tout, de peur d'en pleurer. Après avoir cité à Angran les premiers vers de l'Ode à Dellius d'Horace (souviens-toi que tu vas mourir: "Cette idée suffoque, et applatit tout le reste»), elle s'exclame, toujours pleine d'autodérision : "Mais je m'admire! De vous dire des choses si gaies » (8 août 1753). Entre juillet 1753 et septembre 1755, on voit nettement l'inflexion de la place du rire dans les 35 lettres que la jeune femme adresse à son brillant correspondant. Du début de l'exil en mai 1753, au pardon et au rappel officiel des exilés le $1^{\mathrm{er}}$ septembre 1754, 25 lettres d'Henriette à Angran ont été conservées. Si l'on excepte celles du mois de mai 1754, où Henriette n'a pas le cœur à rire, toutes ces lettres contiennent au moins une information susceptible de faire rire ou sourire son correspondant tout en lui rappelant la culture de leur auteure. Mais, dans les dix lettres suivant le pardon royal, plus de références plaisantes ${ }^{19}$. Cette évolution suit celle de la fréquence de l'écriture épistolaire, qui s'intensifie au cours du printemps et de l'été 1754 : elle passe d'une à deux lettres par mois jusqu'alors, à quasiment une lettre par semaine d'avril à août, et revient ensuite au rythme précédent. Il est manifeste que le retour d'Angran à Paris, en août-septembre 1754, joue un rôle important dans cette évolution, mais elle s'explique aussi par la vie d'Henriette Edme au moment de cet échange épistolaire.

On pourrait avancer ses problèmes de santé, qui lui permettent par ailleurs de valoriser ses connaissances thérapeutiques auprès de l'exilé; mais la politesse et l'honnêteté commandent le rire:

Vous êtes surpris que je conserve de la gayeté au milieu du désastre de ma santé? C’est, Monsieur, si vous ne le sçavez pas un parti très décidé chez moy, d'avoir au moins une écorce dont mes amis puissent s'accommoder, telle peine que j'aye, ou tels maux que je souffre. (2 novembre 1753)

En réalité, ces années représentent un moment charnière dans sa vie. La jeune femme vit avec son beau-père, dont l'âge et la santé laissent prévoir qu' elle sera bientôt sans appui ni protection masculine, situation inenvisageable pour une jeune châtelaine isolée, dans une province reculée. Suivant les lettres à Angran, dès l'été 1754, un des frères d'Henriette, installé à Saint-Domingue, lui propose de venir le rejoindre, ce qu'elle ne peut accepter à cause des "liens ridicules qui [la] tiennent», explique-t-elle le 4 décembre. Mais son second frère, gravement malade, meurt au printemps 1755 . La solution est imposée par son beau-père: elle doit se marier. Les intimes Réflexions journalières d'Henriette, tenues en 1753 et 1754 , nous permettent de suivre ses hésitations et son aspiration à la liberté (Chollet et Krief 2017: 255-324), jusqu'à sa décision finale, qu'expose sa correspondance. En août 1755, elle épouse un cadet de famille car " $[s]$ on beau-père le désire» et elle a été "forcée de prendre un parti», explique-t-elle à Angran dans une lettre sans date.

Durant cette période où pèsent sur elles les attentes de sa famille, le rire «cimente la paire amicale» (Poyet 2013: 192) et devient nécessaire pour affronter l'avenir. Au même titre d'ailleurs que le savoir, seule échappatoire, pourtant interdite. Le rire se mue alors en affrontement du malaise et de la souffrance, «noir» (six occurrences) et «dragons» (8 et 30 août 1753) qu'elle combat par la légèreté. L'épistolière est en lutte contre les normes sociales et la domination masculine. Dans ce contexte,

19. Excepté dans la lettre suivant la confirmation définitive du rappel des parlementaires exilés, et dans la traditionnelle épître du Nouvel An. 
l'écriture, et donc l'échange avec Angran, est un véritable espace de décompression, où Henriette peut défendre son savoir et se dire librement, derrière le rire.

\subsection{Fini de rire?}

Il s'agit donc d'un rire fragile, terriblement dépendant de l'appréciation du correspondant et des aléas de la vie; fragile comme peut l'être l'amitié entre un homme et une femme au regard des normes du siècle $^{20}$, auxquelles, on l'a vu, Henriette accorde de l'importance.

C'est pourquoi la relation épistolaire d'Henriette avec Angran revêt une dimension intéressée. Elle s'y met en valeur, intellectuellement parlant, avec plaisir, parce qu'il est un correspondant avantageux à cultiver. Le rire érudit et aristocratique que met en place Henriette dès les débuts de la correspondance n'est pas uniquement au service de la promotion de soi, il a aussi pour but de créer un lien amical exclusif. Elle évoque ainsi le 29 septembre 1753 «cette attraction qui vous détermine à m'écrire plutôt qu'à une autre"; tandis que, pour sa part, elle affirme: "Je n'en ai bientôt plus de suivi [de commerce de lettres], avec aucun homme excepté vous" ( $1^{\text {er }}$ janvier 1755). En parallèle, elle manipule le rire afin de mieux s'attacher Angran, intermédiaire utile autant qu'aimable dans sa quête de savoirs. Il lui conseille des livres (6 février 1754) et il lui en prête ou en commande ${ }^{21}$. Grâce à lui, elle obtient à la source des nouvelles du conflit entre le Parlement et le roi, qu'elle peut diffuser ensuite à son entourage. Elle acquiert même un rôle actif dans l'activité intellectuelle du parlementaire, probablement privé de ses contacts réguliers par son exil, puisqu'Angran lui fait lire ses textes : "Cela mériterait d'être publié», l'assure-t-elle le 6 octobre 1754. Henriette prend cette tâche au sérieux ("Je m’en suis occupée tout le jour»), car elle peut à travers elle se valoriser, elle qui est une femme seule, non mariée, moins fortunée que ses voisins et d'un rang inférieur à eux: belle stratégie de mise en valeur dans son réseau de sociabilité, qui lui permet de jouer le rôle important de relais de l'information politique la plus récente. En somme, elle bénéfice de cet échange épistolaire non seulement par la circulation des savoirs qu'il implique, mais par l'avantageuse redistribution des rôles qu'il lui procure.

Alors, quand elle envisage la fin de l'exil d'Angran, il n'y a plus de quoi rire: "Nous causerons bien différament, après votre retour, et puis que sçait-on? Il faudrait peu de chose pour nous séparer à jamais, cette perspective n'est pas réjouissante» (6 février 1754). "Vous allez à Paris, où je ne vous verrai pas plus qu'à Bourges, et d'où vous m'écrirez plus difficilement, ainsi touttes les privations vont se réunir", déplore-t-elle le 14 août 1754. Le rappel des exilés signe pour cette femme la fin de la parenthèse érudite et égalitaire, et, de ce fait, marque le retour au "principe de réalité» (Faure 1994: v), car Henriette mène un combat qu'elle ne peut gagner seule. De plus, la cohabitation avec son beau-père est peu plaisante au quotidien : "Vous sçavez à qui, et comme je tiens, je ne dispose pas d'un instant", rappelle-t-elle le 7 août 1754 à Angran qui lui a proposé de le rejoindre. Il est donc peu étonnant qu'au moment où Angran quitte Bourges, la vie quotidienne devienne franchement insupportable pour cette cartésienne: en plein été, son beau-père «se chauffe de son mieux, [...] [elle] en [a] la tête à l'envers, [...] [elle] n'en sçaurai[t] plus rire» (24 août 1754). C'est pourquoi, environ à la même période, elle décide irrévocablement de son avenir: "Tout est dit. Tout est decidé. Il faut voir clair, et prendre son parti, cest dans mon propre courage, quil me faut trouver les ressources dont jai besoin " (Chollet et Krief 2017: 297). L'espace de liberté que représentait l'échange érudit avec Angran se referme, fini de

20. «Cela est rare \& difficile», écrit par exemple $\mathrm{M}^{\mathrm{me}}$ de Lambert (Traité de l'amitié: 37).

21. Henriette a griffonné: «Demander à Mr Angran Velli» (c'est-à-dire l'Histoire de France de l'abbé Velly) sur une lettre du 13 juin 1777 écrite par sa belle-sœur créole et qui ne lui était pas adressée (1Mi3 R18). 
rire. Désormais, la jeune femme garde ses savoirs pour elle et pour son cahier, la lettre n’a plus à être érudite.

Les lettres qu'Henriette Edme écrit à Louis Angran sont l'occasion de valoriser de manière stratégique l'étendue de sa culture et son usage protéiforme du rire. L'épistolière fait intervenir en effet plusieurs types de rire et orchestre leur interaction avec les savoirs qu'elle expose dans ses lettres: un rire bienséant de convention, qui l'inclut, un rire savant qui crée une complicité unique, un rire qui l'isole dans sa singularité d'érudite. La jeune femme ne maîtrise certes pas le rire dévastateur de Voltaire, qui manie badinage, burlesque, ironie et absurde avec virtuosité (Debailly et al. 1994), mais elle témoigne d'un rire féminin et érudit, qui enrichit son discours épistolaire et n’attend pas la Révolution pour être «moderne» (Vaillant et de Villeneuve 2013) : hybride dans sa forme, à la fois honnête et contestataire, léger et sérieux, stratégique et émancipateur.

À la fin de cet épisode, comme on vient de le voir, la demoiselle Edme cède aux pressions extérieures et accepte de se marier, devenant alors $\mathrm{M}^{\mathrm{me}}$ de Marans. Toutefois, elle ne rend pas les armes: elle poursuit son activité d'écriture, allant même jusqu'à publier en 1758, anonymement, les Pensées errantes, fruit de ses réflexions personnelles. Mariage et anonymat confirment le choix d'Henriette de faire preuve d'un apparent conformisme, afin de satisfaire l'opinion publique. Le commerce de lettres avec Angran se poursuit, mais leurs missives n’ont pas été conservées; et les lettres ultérieures de $\mathrm{M}^{\mathrm{me}}$ de Marans dont nous disposons ne revêtent pas de véritable dimension érudite, car elles sont adressées à sa nièce et relèvent de l'écriture domestique quotidienne. Mais, en son for privé, la réflexion continue. Les Confidences générales et particulières, son manuscrit pour les années 1758-1765, sont l'occasion pour Henriette de développer son arsenal: absurde, ironie, animalisation, goguenardise et caricature enrichissent sa plume, libérée des conventions. Et, désormais, le rire et l'érudition féminine sont les armes d'une critique sociale désabusée. Henriette Edme a su mettre à profit un moment grave de la vie de son ami Louis Angran pour faire de ce qui aurait pu être une simple correspondance mondaine un échange au service de son érudition. Sans bouleverser profondément les codes de la lettre familière, elle crée pourtant un espace inédit où promouvoir un «gai savoir» pour les femmes.

\section{Références bibliographiques}

1Mi3 R18. 1777. Archives Départementales de la Sarthe: Le Mans.

Andries, L. 2000. «État des recherches». Dix-Huitième Siècle 32: Le rire, sous la direction de L. Andries: 7-18. "Article LXXX. Nouvelles Littéraires». 1758. Dans Mémoires pour L'Histoire des Sciences et Beaux Arts, commencés d'être imprimés l'an 1701 à Trévoux, \& dédiés à son Altesse Sérénissime Monseigneur le Prince Souverain de Dombes (juillet) : 1897-1918.

Baecque, A. de. 1997. «Les Éclats du rire. Le Régiment de la calotte, ou les stratégies aristocratiques de la gaieté française (1702-1752)». Annales. Histoire, Sciences Sociales. 52 (3): 477-511.

Baecque, A. de. 2000. Les Éclats du rire: La culture des rieurs au XVIII siècle. Paris: Calmann-Lévy.

Boulard, N. 20 juillet 1801. Inventaire après décès de Louis-Alexandre Angran, AN/MC/ET/LXXIII/1 167. Archives Nationales: Paris.

Bourdieu, P. 1979. La Distinction: Critique sociale du jugement. Paris: Éditions de Minuit.

Bray, B. 2001. "La Passion par lettres: Julie de Lespinasse». Dans Conceptualiser la femme dans la pensée des Lumières, sous la direction de H.-E. Bödeker et L. Steinbrügge. Berlin: Arno Spitz: 39-57.

Chollet, M. 2016. Une Ambition de femme au siècle des Lumières. Rennes: Presses Universitaires de Rennes.

Chollet, M. et H. Krief. 2017. Une Femme d'encre et de papier à l'époque des Lumières. Henriette de Marans (1719-1784): Avec l'édition critique de ses journaux manuscrits et inédits (1752-v. 1765). Rennes: Presses Universitaires de Rennes. 
Cotoni, M.-H. 1994. «Le Rire dans la correspondance de Voltaire. L’année 1766 : esprit, ironie, humour ». Dans Rires et sourires littéraires, sous la direction de A. Faure. Nice: Association des publications de la Faculté des lettres de Nice: 111-131.

Debailly, P., J.-J. Robrieux et J. Van den Heuvel. 1994. Le Rire de Voltaire. Paris: Éditions du Félin.

Diaz, B. 1998. «Les Femmes à l'école des lettres. La lettre et l'éducation des femmes au XviII siècle». Dans L'Épistolaire, un genre féminin?, sous la direction de C. Planté. Paris: Champion: 133-150.

Diderot, D. 1875. Réflexions sur Térence. Dans Euvres complètes, vol. 5. Édition préparée par J. Assézat. Paris: Garnier [1762]: 228-238.

Edme des Rouaudières, H. 1753-1755. Lettres à Louis-Alexandre Angran d'Alleray, 1Mi3 R11. Archives Départementales de la Sarthe: Le Mans.

Faure, A. 1994. «Avant-propos. Rire ou comique?». Dans Rires et sourires littéraires, sous la direction de A. Faure. Nice: Association des publications de la Faculté des lettres de Nice: i-ix.

Galiani, F. et L. d'Épinay. 1993. Correspondance II: 1771-février 1772. Édition préparée par G. Dulac et D. Maggetti. Paris: Desjonquères.

Grassi, M.-C. 1981. "Un révélateur de l'éducation au XVIII ${ }^{\mathrm{e}}$ siècle: expressions de la vie affective et correspondances intimes». Revue d'Histoire Moderne et Contemporaine 28 (1): 174-184.

Haroche-Bouzinac, G. 1995. L'Épistolaire. Paris: Hachette.

Lambert, A.-T. de. 1748. Traité de l'amitié. Dans Euvres de Madame la marquise de Lambert, avec un abrégé de sa vie, vol. 1. Paris: Ganeau: 3-40.

Le Goff, J. 1997. "Une Enquête sur le rire». Annales. Histoire, Sciences Sociales 52 (3) : 449-455.

Leroy, N. 7 mai 1733. Inventaire après décès des biens de Louis-Euverte Angran, 3E52/158. Archives Départementales du Loir-et-Cher: Blois.

Maire, C. 2010. «Le Paige et Montesquieu à l'épreuve du vocabulaire des enragés de Bourges». Dans Le Monde parlementaire au XVIII siècle: L’invention d'un discours politique, sous la direction de A. J. Lemaître. Rennes: Presses Universitaires de Rennes: 169-191.

Mallet, E. 1753. Essai sur les bienséances oratoires. 2 vol. Paris: Prault fils.

Nietzsche, F. 2007. Le Gai savoir. Traduit et présenté par P. Wotling. Paris: Gallimard [1882].

Pillet, E. 2000. «Présentation». Humoresques 11 : Armées d'humour. Rires au féminin, sous la direction de J. StoraSandor et E. Pillet: 5-13.

Poirot-Delpech, B. 1994. "Préface». Dans Le Rire de Voltaire, sous la direction de P. Debailly, J.-J. Robrieux et J. Van den Heuvel. 1994. Paris: Éditions du Félin: 9-12.

Pomeau, R. 1971. L'Âge classique III: 1680-1720. Paris: Arthaud.

Poyet, T. 2013. «Le Rire épistolaire: un rire de contradictions. L’exemple Flaubert». Dans Le Rire moderne, sous la direction de A. Vaillant et R. de Villeneuve. Paris: Presses Universitaires de Paris Ouest: 189-201.

Rauline, L. 2007. «Le Voyage de Chapelle et Bachaumont: les itinéraires du divertissement ou les voies obliques de la critique politique». Dans Le Rire des voyageurs (XVI -XVIT siècles), sous la direction de D. Bertrand. Clermont-Ferrand: Presses Universitaires Blaise Pascal : 107-124.

Reynolds, S. 2010. «Medusa laughs; le rire féministe». Dans Le Rire européen, sous la direction d'A. Chamayou et A. B. Duncan. Perpignan: Presses Universitaires de Perpignan: 293-303.

Richardot, A. 2002. Le Rire des Lumières. Paris: Champion.

Riel, F. 18 août 1755. État et mémoire des meubles [...] sur la terre des Rouaudières, 1Mi3R11. Archives Départementales de la Sarthe: Le Mans.

Vaillant, A. et R. de Villeneuve, dir. 2013. Le Rire moderne. Paris: Presses Universitaires de Paris Ouest.

Vaugelas, C. F. de. 2009. Remarques sur la langue françoise. Édité par Z. Marzys. Genève: Droz [1647].

Velly, P. F. 1770. Histoire de France, depuis l'établissement de la monarchie jusqu'à Louis XIV. Paris: Saillant, Nyon et Desaint. 
Whatley, J. 1997. «Letters to a Libertine: the Correspondence of Belle de Zuylen and Constant d'Hermenches». Dans Women Writers in Pre-Revolutionary France: Strategies of Emancipation, sous la direction de C. H. Winn et D. Kuizenga. New York: Garland Publishing: 335-348. 